\title{
Synchronization and Stability in Noisy Population Dynamics
}

\author{
Sabrina B.L. Araujo and M.A.M. de Aguiar \\ Instituto de Física 'Gleb Wataghin', Universidade Estadual de Campinas, \\ Caixa Postal 6165, 13083-970 Campinas, São Paulo, Brazil
}

\begin{abstract}
We study the stability and synchronization of predator-prey populations subjected to noise. The system is described by patches of local populations coupled by migration and predation over a neighborhood. When a single patch is considered, random perturbations tend to destabilize the populations, leading to extinction. If the number of patches is small, stabilization in the presence of noise is maintained at the expense of synchronization. As the number of patches increases, both the stability and the synchrony among patches increase. However, a residual asynchrony, large compared with the noise amplitude, seems to persist even in the limit of infinite number of patches. Therefore, the mechanism of stabilization by asynchrony recently proposed by R. Abta et. al. [7], combining noise, diffusion and nonlinearities, seems to be more general than first proposed.
\end{abstract}

PACS numbers: 87.23.Cc,05.45.Xt,87.18.Hf

The model proposed independently by Lotka [1] and Volterra 2] was probably the first to describe mathematically the dynamics of predators and preys. Its success and widespread use by early biologists is mostly due to its ability to qualitatively describe the population oscillations of both preys and predators [3]. The model, however, is well known to be unstable under the addition of noise, which causes the amplitude of the population oscillations to increase until one (or both) species eventually becomes extinct. Stability, in the sense of co-existence of both species, can be regained if several patches of populations are coupled via dispersal or predation. Computational simulations have shown that stability increases (with respect to noise amplitude) with the number of patches considered [4, 5, 6]. The ultimate reason for the stabilization was recently pinned down by R. Abta et. al. [7], who studied in detail the case of two patches. They concluded that the crucial condition for stabilization is the development of an asynchrony between the population oscillations in each patch, resulting from the combined action of diffusion and noise. Such an asynchrony develops if the frequency of the population oscillations depends on their amplitude.

Although the results of ref. [7] are of theoretical and conceptual importance, simple Lotka-Volterra equations (LV, for short) are seldom used to model population dynamics now-a-days. Instead, models involving logistic type of interactions displaying limit cycles or chaotic attractors have become common [9, 10, 11, 12]. In this report we discuss the problem of stabilization and synchronization for a predator-prey system displaying an attracting limit cycle [8]. For this system all asymptotic orbits have the same oscillation frequency, which is the frequency of the attractor. As a consequence, orbits displaced from the attractor by a perturbation will return to the attractor and to the same frequency of oscillation, different from LV model. We shall call this system LC (limit cycle) for short. The system is only weakly stable in the presence of noise, in the sense that it becomes unstable when the noise amplitude crosses a threshold which is very small. Therefore, for noise amplitudes that are not too small, LC behaves like LV, with noise driving one or both species to extinction.

A spatial version of the LC model, SLC, can be constructed by allowing patches of local populations to interact. If the patches are strongly coupled, the dynamics in each spatial region synchronizes and the system behaves like a single well mixed population, identical to the original LC model. Here we study the stability and synchronization of the spatial model under random perturbations in the strongly coupled regime. Because of the attracting limit cycle, it is not clear that an asynchrony among patches will develop, since perturbed orbits will always have similar amplitudes and, therefore, similar oscillation frequencies. For a system with only two patches, we show that desynchronization indeed takes place, leading to the stabilization of the population oscillations, in agreement with the results of [7] for the LV model. As more patches are added, SLC system becomes stable under larger noise amplitudes [4] and the asynchrony decreases exponentially with system size. However, a residual asynchrony, much larger than the noise amplitude, seems to survive even in the limit of infinite number of patches. Therefore, even for large systems displaying an attracting limit cycle, the combined action of diffusion and noise still plays a crucial role in desynchronizing the patches.

The LC predator-prey model with noise is given by the equations

$$
\begin{aligned}
& x_{n+1}=\left[\frac{x_{n}}{x_{n}(1-a)+a}\right] P_{x}\left(y_{n}\right)+\eta_{x}, \\
& y_{n+1}=y_{n}\left[e^{-d_{1}}+F_{y}\left(x_{n}\right)\right]+\eta_{y}
\end{aligned}
$$

where $P_{x}(y)=e^{-y / \alpha}$ accounts for the predation of $y$ upon the prey $x$ and $F_{y}(x)=1-e^{-x / \beta}$ for the reproduction of $y$ due to the feeding upon $x$. The variables $\eta_{x}$ and $\eta_{y}$ are random numbers representing the external noise whose distributions are homogeneous and limited to the interval $[-\Delta, \Delta]$.

To understand the role of each term in these equations let us first consider $\eta_{x}=\eta_{y}=0$. Then, in the absence 
of predators, $P_{x}(0)=1$ and the population of preys converges to the normalized value $x=1$, provided $a<1$. As the number of predators increases, $P_{x}(y)$ decreases, reducing the population of preys. Similarly, in the absence of preys $F_{y}(0)=0$ and the population of predators decreases steadily because of the intrinsic death rate $d_{1}$. When the number of preys is sufficiently large so that $e^{-d_{1}}+F_{y}(x)>1$ the number of births becomes larger than the number of deaths and the population of predators grows.

Following [8] we fix the model parameters at $a=e^{-1}$, $\alpha=0.8$ and $\beta=0.4$. In the absence of noise the system displays two kinds of attractive orbits, depending on the value of the death rate $d_{1}$ : for $0<d_{1}<0.6$ the attractor is a limit cycle and for $d_{1}>0.6$ it is a fixed point. When the noise is turned on it might happen that the population densities $x$ or $y$ become less than zero. In this case we set it back to zero to avoid negative values.

Throughout this paper we have fixed the death rate of the predator at $d_{1}=0.1$. In this case the trajectories converge to a limit cycle where the prey population oscillates between $2.7 \times 10^{-4}$ and 0.3 . This attractor is only very weakly stable, since the addition of noise with amplitudes of the order of $\Delta=6 \times 10^{-5}$ is enough to drive both species to extinction.

To consider more than one patch we extend the LC model to [8]:

$$
\begin{aligned}
x_{n+1}^{i, j}= & {\left[\frac{x_{n}^{i, j}}{x_{n}^{i, j}(1-a)+a}\right]\left\langle P_{x}\left(y_{n}\right)\right\rangle_{R} } \\
& +\frac{m_{x}}{4}\left(\sum_{l, m} x^{i+l, j+m}\right)-m_{x} x^{i, j}+\eta_{x_{i, j}} \\
y_{n+1}^{i, j}= & y_{n}^{i, j}\left[e^{-d_{1}}+F_{y}\left(\left\langle x_{n}\right\rangle\right)\right] \\
& +\frac{m_{y}}{4}\left(\sum_{l, m} y^{i+l, j+m}\right)-m_{y} y^{i, j}+\eta_{y_{i, j}}
\end{aligned}
$$

where $i, j$ label the position of the patches on a twodimensional grid of $N \times N$ patches. This spatial version (SLC) allows migration of predators and preys, with rates $m_{y}$ and $m_{x}$ respectively, and predation over a predation neighborhood $R$. Here we consider square neighborhoods with sides $2 R+1$ centered on the predator.

The sum over $l$ and $m$ in the migration terms is restricted only to the four nearest neighbors of the site $(i, j)$. The averages $\left\langle P_{x}\left(y_{n}\right)\right\rangle_{R}$ are performed over the patches that are in predation neighborhood: $\left\langle P_{x}^{i, j}(y)\right\rangle_{R}=\frac{1}{N_{R}} \sum_{l, m=-R}^{R} P_{x}\left(y^{i+l, j+m}\right)$ where $N_{R}=$ $(2 R+1)^{2}$ is the number of patches within the neighborhood. The feeding function $F_{y}\left(\langle x\rangle_{R^{i, j}}\right)$ is calculated over the average number of $x$ on the predation neighborhood: $F_{y}\left(\langle x\rangle_{R}\right)=F_{y}\left(\frac{1}{N_{R}} \sum_{l, m=-R}^{R} x^{i+l, j+m}\right)$. We fix the migration rate of the prey and the predator at $m_{x}=0.01$ and $m_{y}=0.1$ respectively, so that the migration rate of the predator is larger then that of the prey [13, 14].
In the SLC model, the average dynamic behavior can be very different from that of the LC model. However, if the predation neighborhood encompasses most of the patches in the grid, the system becomes strongly coupled and the time evolution in each patch synchronizes with the others [8].

\section{Two patches}

In our first analysis we consider only two patches. In this case Eq.(2) reduces to

$$
\begin{aligned}
x_{n+1}^{i}= & {\left[\frac{x_{n}^{i}}{x_{n}^{i}(1-a)+a}\right]\left\langle P_{x}\left(y_{n}\right)\right\rangle } \\
& +m_{x} x^{j}-m_{x} x^{i}+\eta_{x_{i}} \\
y_{n+1}^{i}= & y_{n}^{i}\left[e^{-d_{1}}+F_{y}\left(\left\langle x_{n}\right\rangle\right)\right]+m_{y} y^{j} \\
& -m_{y} y^{i}+\eta_{y_{i}} .
\end{aligned}
$$

where $i$ labels one of the patches and $j$ the other one. The averages are given by $\left\langle P_{x}\left(y_{n}\right)\right\rangle=\frac{1}{2}\left(P_{x}\left(y^{i}\right)+P_{x}\left(y^{j}\right)\right)$ and $F_{y}\left(\left\langle x_{n}\right\rangle\right)=F_{y}\left(\frac{x^{i}+x^{j}}{2}\right)$. In the absence of noise and with random initial conditions, the time evolution of the populations synchronize perfectly. However, when noise is added to the system, the populations oscillate between synchronized and desynchronized phases. In our simulations we let the system evolve without noise for 2,000 time steps, which is enough to synchronize the patches. Noise with amplitude of $\Delta=6 \times 10^{-5}$ (which is enough to destabilize the dynamics on a single patch) is then added to the system, which is further evolved for another 28,000 steps. Figure 1 shows the populations in one of the patches in the phase-space for times between 1,000 and 10,000 . The coupling between the patches stabilizes the system. For larger noise amplitudes, of the order of $\Delta=2 \times 10^{-4}$, instability sets in again and both species may go extinct.

In order to quantify the synchrony between the patches, we fixed a reference point at $\overrightarrow{r_{0}}=(0.04,0.75)$ and measured the phase and amplitude differences between the vectors $\vec{r}_{01}=\overrightarrow{r_{0}}-\vec{r}_{1} \equiv r_{01} e^{i \theta_{01}}$ and $\vec{r}_{02}=\overrightarrow{r_{0}}-\vec{r}_{2} \equiv r_{02} e^{i \theta_{02}}$, where $\vec{r}_{i}$ is the phase space position of the populations in patch $i$. Figure 2 shows the phase difference $\left|\theta_{01}-\theta_{02}\right|$ and amplitude difference $\left|r_{01}-r_{02}\right|$. As the initial conditions for each patch are different, the phase and amplitude differences are initially non-zero. However, the patches quickly synchronize in the absence of noise (first 2,000 time steps) and de-synchronize again when noise is added.

\section{Increasing the number of patches}

When more patches are taken into account, the stability properties change qualitatively. We considered the spatial model on a set of $N \times N$ patches, Eq. (2), with periodic boundary conditions. As before, we fixed $d_{1}=0.1$, $\alpha=0.8, \alpha=0.4, m_{x}=0.01, m_{y}=0.1$ and $\Delta=6 \times 10^{-5}$. 


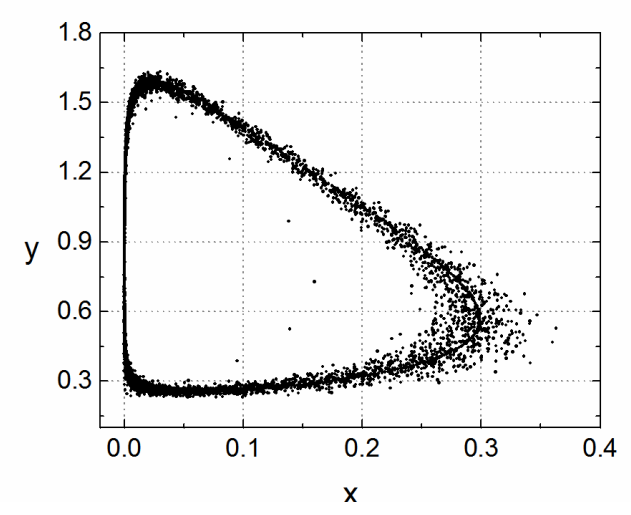

Figure 1: Phase-space trajectories for the case of two patches. The plots show the time steps between 1,000 and 10,000 for only one patch. The patches are synchronized up to 2,000 steps, when noise is added and synchronization breaks down. The patches nearly synchronize when $x \approx 0$ and get significantly desynchronized for large $x$.

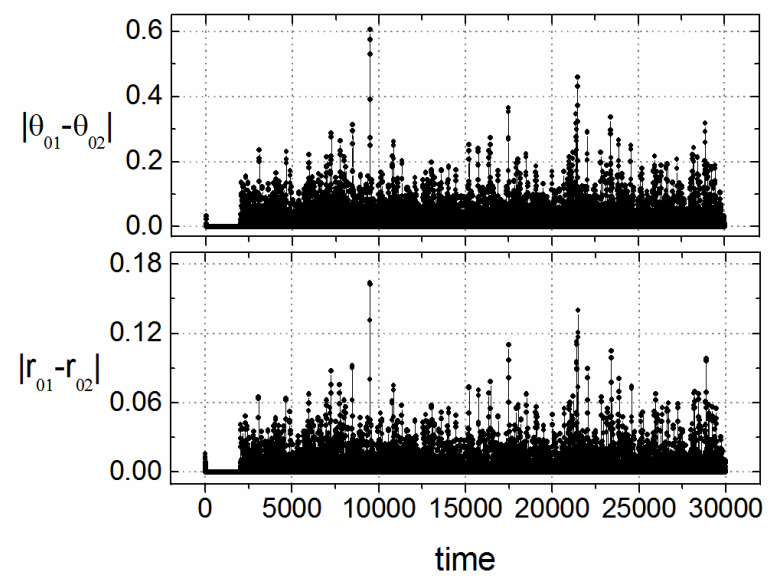

Figure 2: Phase (in radians) and amplitude differences between the two patches for the trajectories shown in Figure (1). The crests correspond to regions where $x$ is large.

The only free parameters are the sizes of the grid, $N$, and the predation radius, $R$. As in the case of two patches, it is possible to synchronize the patches in the absence of noise if the coupling is sufficiently strong [8].

In order to study the effect of noise on large synchronized systems we did simulations in which $N$ was varied but the ratio $(2 R+1) / N$, was kept constant. We considered 8 combinations of $N$ and $R$ with $(2 R+1) / N=5 / 6$ : (1) $N=6, R=2$; (2) $N=18, R=7$; (3) $N=30$, $R=12$; (4) $N=42, R=17$; (5) $N=54, R=22$; (6) $N=66, R=27 ;(7) N=78, R=32 ;$ (8) $N=90$, $R=37$. In all cases the patches synchronized in the absence of noise.

The simulations started with random initial conditions and were iterated by 60,000 time steps. Noise was added only after the first 5,000 steps. As in the case of two patches, we fixed the reference point $\overrightarrow{r_{0}}=(0.04,0.75)$ and calculated the phase and amplitude differences for each patch with respect to patch number one. The temporal averages of the phase and amplitude differences were computed for each individual patch for the last 50,000 time steps, $\left\langle\left|\theta_{1}-\theta_{i}\right|\right\rangle$ and $\left\langle\left|\vec{r}_{1}-\vec{r}_{i}\right|\right\rangle$ and also the global average, $\Delta \bar{\theta}=\frac{1}{N^{2}-1} \sum_{i=2}^{N^{2}}\left\langle\left|\theta_{1}-\theta_{i}\right|\right\rangle$ and $\Delta \bar{r}=\frac{1}{N^{2}-1} \sum_{i=2}^{N^{2}}\left\langle\left|\vec{r}_{1}-\vec{r}_{i}\right|\right\rangle$.

In agreement with D. D. Donalson et. al. [4] we found that the populations resist to higher noise amplitudes as the number of patches increases. Moreover, for fixed noise amplitude and coupling ratio, the patches tend to become more synchronized as $N$ increases. Therefore, for large systems, stability and asynchrony are not as correlated as in the case of two patches [7].

Figure 3 shows that the average asynchrony decreases exponentially with grid size, following approximately the curve $y(N)=A \exp (-N / b)+c$. For the amplitude difference (gray curve) we found $A=0.017, b=18$ and $c=0.016$ and for the phase difference $A=0.011, b=16$ and $c=0.0046$. In the limit of infinitely many patches the average amplitude difference tends to $c=0.046$, which is significantly larger than the noise, $\Delta=6 \times 10^{-5}$. The conclusion of these numerical experiments is that the

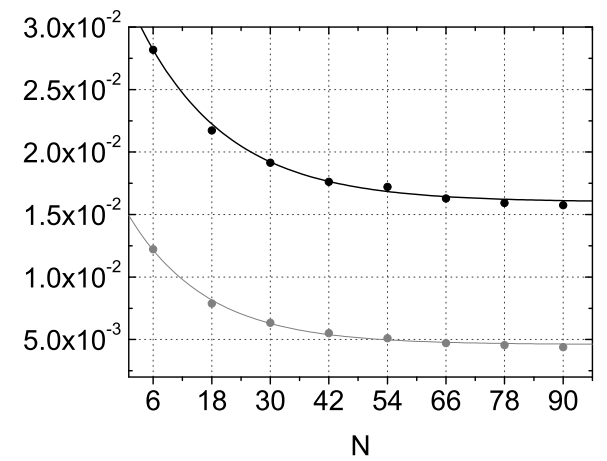

Figure 3: Global average of phase difference $\Delta \bar{\theta}$ (black), and amplitude difference $\Delta \bar{r}$ (gray), as a function of grid size, $N$. The lines are first order exponential decay fit.

simultaneous presence of noise and diffusion seems to lead to significant asynchrony even if the unperturbed dynamics has an attractive limit cycle. For small systems this asynchrony can stabilize the populations by allowing the migration of individuals from more populated patches to those where extinction is imminent. For large systems, asynchrony decreases exponentially fast with system size but never disappears completely.

The mechanism responsible for the desynchronization in systems with attractors seems to be the same proposed in [7], i.e., motion with amplitude depend frequencies. Since the dynamics tend to bring perturbed orbits back to the attractor, a typical trajectory always wanders in the vicinity of the attractor. However, the nonlinear character of the equations amplify these small deviations producing significant frequency differences that are reflected in the desynchronization. Therefore, 
the mechanism of R. Abta et. al. 7], combining noise, diffusion and nonlinearities, seems to be more general than first proposed.
Acknowledgements This work was partially supported by Fapesp and CNPq.
[1] A.J. Lotka Elements of Physical Biology, Baltimore, Williams and Willkins (1925).

[2] V. Volterra, Journal du Conceil, Conceil International pour l'exploration de la Mer, 33 (1928).

[3] J. D. Murray. Mathematical Biology. Springer, Berlin, 1993.

[4] D. D. Donalson and R. M. Nisbet, Ecology. 43, (1999).

[5] W. G. Wilson, A. M. Deroos and E. Mccauley, Theor. Popul. Biol 43, (1993).

[6] C. J. Brigges and M. F. Hoopes, Theor. Popul. Biol 65 , (2004).

[7] R. Abta, M. Schiffer and N. M. Shnerb, Phys. Rev. Letters 98, 098104 (2007).

[8] S. B. L. Araujo and M. A. M. de Aguiar, Phys. Rev. E 75, 061908 (2007).
[9] W. M. Schaffer, The American Naturalist. 124, 798 (1984).

[10] A. Hastings and T. Powell, Ecology. 72, 896 (1991).

[11] A. Hastings, C. L. Hom, S. Ellner, P. Turchin and H. C. J. Godfray, Annual Review of Ecology and Systematics. 24, 1 (1993).

[12] J. Chattopadhyay and R. R. Sarkar, Journal of Ecological Modelling. 163, 45 (2003).

[13] J. Bascopte and R. V. Solé, J. Theor. Bio. 195, 383 (1998).

[14] R. V. Solé and J. Bascopte, Self-Organization in Complex Ecosystems, Monographs in Populatin Biology, Princenton University Press (2006). 